viruses no significant differences in HRV versus controls were observed. These data indicate RSV related reduced sympathetic activity.

Conclusion RSV infection in infants is associated with autonomic dysfunction of central origin. This may be correlated with an increased risk for serious apnea or ALTE, for which prolonged cardio respiratory monitoring is indicated.

\section{ACUTE RESPIRATORY TRACT INFECTIONS IN PRESCHOOL POPULATION OF GEORGIA}

doi:10.1136/archdischild-2012-302724.0942

M Kherkheulidze, N Kavlashvili, I Chkhaidze, E Kandelaki, N Adamia. Pediatrics, State Medical University, Tbilisi, Georgia

Aim and methods: To assess the frequency of ARI in preschool children population, identification of risk factors and main principles of treatment. The cross sectional study was conducted using the special questionnaires for parents of 3-6 years children and in all regions of Georgia. At all 1448 parents and caregivers were interviewed.

Results $2.3 \%$ of children have 7-8 episodes of ARI per year, $19.9 \%$ have $3-5,53.4 \% 1-2$ episodes per year and in $24.2 \%$ ARI are very rare. The incidence of ARI is higher in urban population, then in rural area and in mountain regions $(p<0,001)$. The main symptoms for admission to health care facilities were cough (82, 8\%) and fever $(75,9 \%)$. Study revealed the risk factors for ARI: male sex, living in urban area, attendance of day care centers, exposure to passive smocking, big number of family members significantly increase risk of ARI. There was no correlation between the type of feeding in infancy and frequency of ARI infection in 3-6 years old children. In $47 \%$ of ARI cases antibiotics were used from those in 32 \# parents started antibiotics by self.

Conclusion Male sexes, urban regions, attendance of day care center, passive smoking are the significant risk factors for development of ARI in children aged 3-6 years. Primary health care providers still prescribe unnecessary and excessive antibiotics. The incidence of ARI may be reduced substantially through public health measures.

\section{THE STUDY OF ANTIMICROBIAL RESISTANCE AMONG SHIGELLA FLEXNERI STRAINS ISOLATED OF CHILDREN IN TEHRAN, IRAN}

doi:10.1136/archdischild-2012-302724.0943

'Z Rajabi, ${ }^{2} \mathrm{MM}$ Soltan Dallal, ${ }^{3} \mathrm{MR}$ Pourshafie, ${ }^{4} \mathrm{R}$ Ranjbar, ${ }^{5} \mathrm{~S}$ Heidarzadeh. ${ }^{1}$ Pathobiology, School of Public Health, Tehran University of Medical Sciences; ${ }^{2}$ Pathobiology, School of Public Health, Antibiotic Resistant Research Center, Tehran University of Medical Sciences; ${ }^{3}$ Microbiology, Institute Pasteure of Iran; ${ }^{4}$ Molecular Biology Research Center, Baqiyatallah University of Medical Sciences; ${ }^{5}$ Pathobiology, School of Paramedical Sciences, Tehran University of Medical Sciences, Tehran, Iran

Background and Aims Shigellosis is one of the major causes of morbidity in children with diarrhea in Iran. The aim of this study was to investigate antimicrobial resistance of $S$. flexneri strains isolated from clinically diagnosed cases of gastroenteritis and acute diarrhea in Tehran, Iran.

Methods Shigella strains were isolated from stool samples of children patients who visited the several major hospitals in Tehran. S. flexneri was preliminarily identified by biochemical tests as well as by API20E. Antimicrobial resistance testing was performed according to the standard guidelines of the Clinical and Laboratory Standards Institute.

Results All strains were resistant to streptomycin. More than $97 \%$ of the strains were resistant to tetracycline, $89 \%$ to co-trimoxazole, $80 \%$ to amoxicillin, $33.5 \%$ to ampicillin, $14 \%$ to chloramphenicol, $8 \%$ to kanamycin, $5 \%$ to nalidixic acid, $1.5 \%$ to cefixime and $0.5 \%$ to amikacin and furazolidone. None of the tested isolate was resistant to ceftriaxone, ceftizoxime, ceftazidime, gentamicin, ciprofloxacin cephalothin, cefotaxime, cephalexine and nitrofurantoin.

Conclusions This study indicates the increase in incidence of multiple drug resistance among the strains of $S$. flexneri isolated in Tehran, Iran.

\section{PRECEDING HUMAN METAPNEUMOVIRUS INFECTION INCREASES ADHERENCE OF STREPTOCOCCUS PNEUMONIAE AND SEVERITY OF MURINE PNEUMOCOCCAL PNEUMONIA}

doi:10.1136/archdischild-2012-302724.0944

SH Lai, SL Liao, KS Wong, TY Lin. Pediatrics, Chang Gung Memorial Hospital, Taoyuan Taiwan R.O.C.

Co-infections with respiratory virus and Streptococcus pneumoniae have been frequently reported in epidemiologic studies. Here, we explore how inoculating A549 and Hep-2 cell monolayers with human metapneumovirus (hMPV) affected subsequent in vitro infections with pneumococcus serotypes 3 and 14 . The different serotypes had variable adherence, but the A549 cells with a preceding hMPV infection had significantly enhanced bacterial adherence. Also, BALB/c mice infected with hMPV had increased adhesion of pneumococcus to the bronchial epithelium. The lungs of mice with a preceding hMPV inoculation had delayed bacterial clearance and exacerbated histopathology after they were infected with Streptococcus pneumoniae. Additionally, the mice with a preceding hMPV infection had inhibited recruitment of airway neutrophil and decreased expression of neutrophil chemoattractants. Our results suggest that

1. airways infected with hMPV, especially lower airway epithelium, allow increased adherence of Streptococcus pneumoniae and

2. hMPV-infected mice have impaired recruitment of airway neutrophils that may cause delayed bacterial clearance and exacerbated pulmonary inflammation.

\section{CHANGES OF INTESTINAL SECRETORY IMMUNOGLOBULIN A IN CHILDREN WITH ROTAVIRUS INFECTION}

doi:10.1136/archdischild-2012-302724.0945

I Nezgoda, 0 Bodnariuk. Infectious Dideases in Children, Vinnitsa National Medical M.I. Pirogov Memorial University, Vinnytsya, Ukraine

The Aim of this study was to evaluate the level of intestinal sIgA in children with rotavirus infection (RVI) depending on disease severity.

Methods Between November 2009 and February 2011, stool specimens from 85 children less than 4 years of age suffering from RVI were tested for intestinal sIgA by ELISA. $75.6 \%$ of patients were with moderate severity of disease and $23.5 \%$ - with severe course. 66 healthy children were studied as controls.

Results intestinal $\operatorname{sigA}$ in patients on the $1^{\text {st }}-2^{\text {nd }}$ day of RVI was $22.6 \pm 1.05 \mathrm{mg} / \mathrm{l}$ and significantly different $(\mathrm{p}<0.01)$ from the level of healthy children $31.8 \pm 1.35 \mathrm{mg} / 1$. The following dependence from disease severity was detected: intestinal sIgA in severe case$16.7 \pm 1.6 \mathrm{mg} / \mathrm{l}$ which was significantly less $(\mathrm{p}<0,001)$ than in patients with moderate severity $24.36 \pm 1.21 \mathrm{mg} / 1$. Intestinal sIg A increased up to $32.74 \pm 1.53 \mathrm{mg} / \mathrm{l}$ in patients with moderate severity on the $5^{\text {th }}$ $6^{\text {th }}$ day of disease and to $22.9 \pm 1.85 \mathrm{mg} / \mathrm{l}$ in patients with severe case which were significantly different $(\mathrm{p}<0.05)$.

Conclusions The level of intestinal sIgA on the $1^{\text {st }}-2^{\text {nd }}$ day of disease was less than the level of healthy children. Severe course of disease is usually present in case of low concentration of sIgA. The level of sIgA in patients with moderate severity of RVI increases up to the level of healthy children on the $5^{\text {th }}-6^{\text {th }}$ day of disease and it is significantly higher than the level of sIgA in patients with severe 